\title{
Cantharidin: An Active Compound of Blister Beetle Caused Mitochondrial Damage and Induced Apoptosis, Necrosis and Autophagy in Dalton's Ascites Lymphoma in vivo
}

\author{
Akalesh K. Verma ${ }^{1,2}$ and Surya B. Prasad ${ }^{1}$ \\ ${ }^{1}$ Department of Zoology, Cell and Tumor Biology Laboratory, North-Eastern Hill University, \\ Shillong-793 022, India \\ ${ }^{2}$ Cachar Cancer Hospital \& Research Centre, Meherpur, Silchar- 788015, India
}

Prevention is undeniably the sensible maneuver towards the ultimate goal of cancer control [1]. According to the World Cancer Report released by the World Health Organization (WHO) in 2003 [2], cancer rates could further increase by $50 \%$ to 15 million new cases in the year 2020. Chemotherapy, radiotherapy and surgery are commonly used treatment methods for cancer in modern medicine. Chemotherapy is now considered as the most effective method of cancer treatment. However, most cancer chemotheraputants severely affect the host normal cells [3]. Hence, the use of natural products mainly plants and animals now have been contemplated of exceptional value in the control of cancer and its eradication program [1].

Cantharidin, a type of terpenoid, is a blistering agent of most of the blister beetles. The isolation and antitumor activity of cantharidin from blister beetles, Mylabris cichorii has been recently reported by us [4]. This study aim at to study the ultrastructural changes in mitochondria with their function and understanding their significance in the underlying mechanism associated with cantharidin-mediated antitumor effects in Dalton's lymphoma (DL) bearing mice which is not yet reported.

Transmission electron microscopic (TEM) study after cantharidin treatment $(0.5 \mathrm{mg} / \mathrm{kg})$ in DL bearing mice caused the appearance of abnormal mitochondrial features (Fig. 1) which included roundish mitochondria with thickened membranes, irregularity in cristae, and appearance of small to large size vacuoles in mitochondria of DL cells. As compared with the corresponding control, cantharidin-treated mice also showed a significant time dependent decrease in succinate dehydrogenase (SDH) activity in the mitochondria of DL cells moreover, molecular docking study also showed strong interaction with cantharidin in SDH active site (Fig 4). The ampholytic cationic fluorescent probe rhodamine-123 was used to monitor cantharidin-induced changes in the mitochondrial transmembrane potential using flow cytometry and the result showed cantharidin-mediated time-dependent shifting of mitochondrial fluorescence intensity toward the left indicating a decrease in mitochondrial membrane potential (Fig. $3)$. Cantharidin treatment also induced apoptosis (69\%), necrosis $(8 \%)$ and autophagy (12\%) in DL cells after $96 \mathrm{~h}$ of treatment (Fig. 2). The apoptotic morphological features using confocal microscopy based study reflects cell shrinkage, cytoplasmic condensation, DNA fragmentation and membrane blebbing; whereas necrosis involves loss of membrane integrity, cell swelling, formation of cytoplasmic vacuoles, swollen endoplasmic reticulum and ruptured mitochondria while autophagy cells shows the formation of autophagosomes that engulf cellular macromolecules and organelles, leading to their breakdown, following fusion with lysosomes. Flow cytometry-based cell cycle analysis showed a time dependent accumulation of the sub-G0 population of DL cells, thus, confirming the involvement of apoptosis in tumor cells in cantharidin-mediated antitumor activity. Based on the result of present study it may be suggested that these cantharidin-mediated changes in mitochondrial structure and function may play a crucial role in its antitumor activity. 


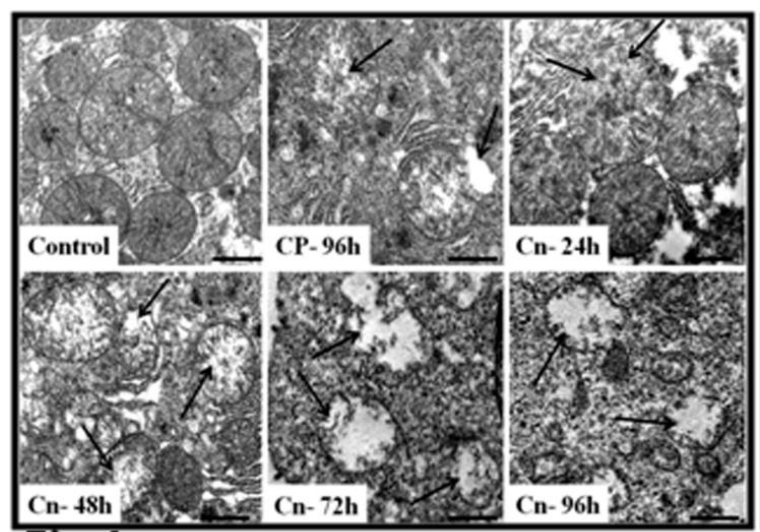

Fig 1

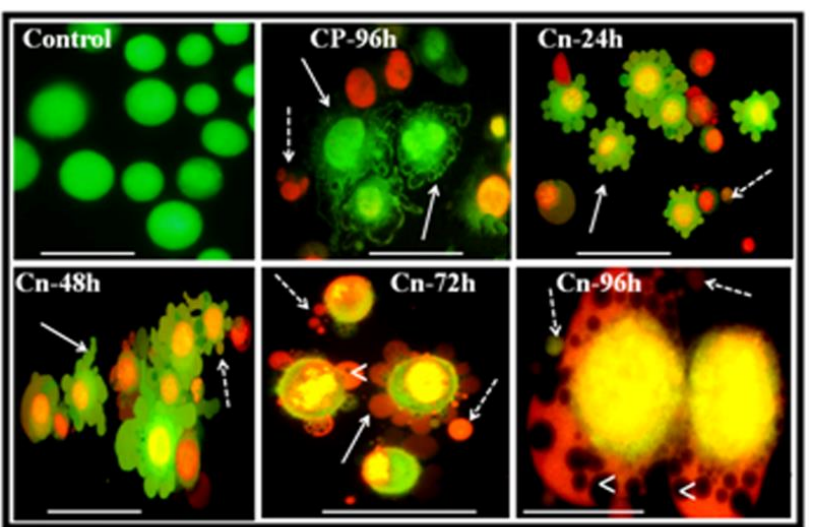

Fig 2

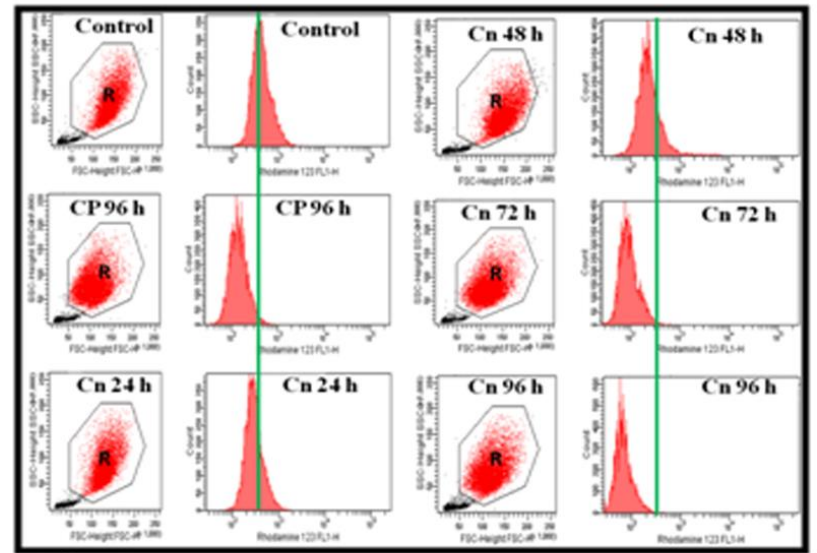

Fig 3

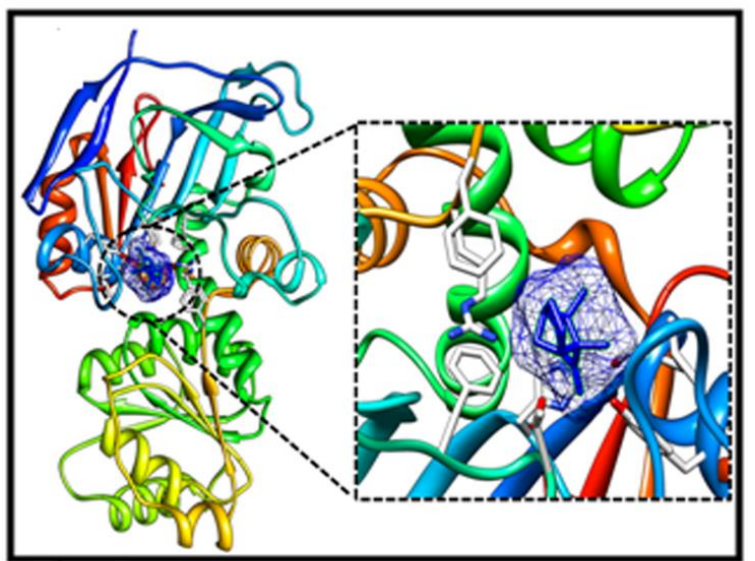

Fig 4

Figure 1 Showing ultrastructural changes in mitochondria after cantharidin treatment, arrow shows mitochondrial damage, scale- $1 \mu \mathrm{m}$; Figure 2 shows cantharidin-mediated severe apoptotic features in DL cells, regular arrow shows membrane blebbing, dotted arrow shows apoptotic bodies and arrowhead shows vacuoles, scale- $20 \mu \mathrm{m}$; Figure 3 showing decrease in mitochondrial membrane potential (both dots plot and peak are shown for each treatment group) and Figure $\mathbf{4}$ showing interaction of succinate dehydrogenase with cantharidin which may caused inhibition of this enzyme. Cn- cantharidin $(0.5 \mathrm{mg} / \mathrm{kg}), \mathrm{Cp}$ - cisplatin (reference drug, $2 \mathrm{mg} / \mathrm{kg}$ ) and R-Rhodamine- 123 .

\section{References}

1. Suffness, M and Pezzuto, JM in "Methods in Plant Biochemistry", ed. Hostettmann K, (Academic Press, New York) 1991, p. 71-134.

2. World Health Organization (WHO) (2008). Traditional Medicine Fact Sheet No. 134. Available at www.who.int/mediacentre /factsheets/fs134/en/.

3. Mascarenhas, M, Mushroom Research 3 (1994), 77-80.

4. Verma, AK and Prasad, SB, Cell Biology and Toxicology 28 (2012), 133-147.

5. The authors acknowledge the funding from University Grant Commission, New Delhi India, (F-4-1/2006(BSR)/5-120/2007(BSR), Dated 20 ${ }^{\text {th }}$ January'09. 